\title{
Die Viszeralchirurgie ermöglicht Hoffnung beim Pankreaskarzinom
}

\author{
Lukasz Filip Grocholaa, Stefan Breitenstein ${ }^{b}$ \\ ${ }^{a}$ Dr. med., PhD, Departement Chirurgie, Kantonsspital Winterthur; ${ }^{b}$ Prof. Dr. med., Departement Chirurgie, Kantonsspital Winterthur
}

Die Viszeralchirurgie als Fachgebiet der insbesondere auf die Bauchorgane spezialisierten Chirurginnen und Chirurgen hat in den letzten Jahrzehnten nennenswerte Fortschritte gemacht. Vor allem im Bereich der Operationstechniken haben sich mit vermehrtem Einsatz der Laparoskopie Verbesserungen für die Patienten ergeben. Auch bei Erkrankungen wie dem Pankreaskarzinom mit nach wie vor ernüchternder Prognose sind Fortschritte zu verzeichnen.

Das Kosten-Nutzen-Verhältnis der medizinischen Versorgung wird längst auch im Bereich der Viszeralchirurgie im Rahmen eines öffentlichen sowie wissenschaftlichen Diskurses stark debattiert. Diese sowohl innerhalb der Schweiz wie auch auf internationaler Ebene geführte Debatte hat bereits in den letzten Jahren wichtige Optimierungsprozesse in diesem Fachgebiet angestossen. So wird die Verlagerung stationärer Eingriffe in den teilstationären und ambulanten Bereich gezielt angegangen. Aber auch die sich in den vergangenen Jahrzehnten zunehmend verbreitenden laparoskopischen Techniken, bei denen mit Hilfe einer in den Bauchraum eingebrachten Videokamera minimal-invasiv operiert werden kann, werden unter diesem Aspekt kritisch und genau analysiert. Auf der einen Seite ist die Laparoskopie mit höheren Materialkosten verbunden, auf der anderen Seite wird jedoch die Erholung nach der Operation verbessert und die Zeit im Spital verkürzt, was wiederum die Gesamtkosten senkt. Nicht zuletzt haben Kosten-Nutzen-Analysen inzwischen zu einer breiten Akzeptanz dieser Technik geführt [1]. ${ }^{1}$

Herr S., ein 37-jähriger Monteur, leidet an schmerzhaften beidseitigen Leistenbrüchen, welche ihn bei der Arbeit beeinträchtigen. Es wird eine minimal-invasive operative Versorgung beider Hernien mit Implantation von gut gewebeverträglichen Netzen über insgesamt drei kleine (1-3 cm grosse) Schnitte gemacht. Der gesamte Eingriff dauert weniger als 90 Minuten und ist für den Patienten nur wenig belastend, so dass Herr S. schon am Folgetag das Spital verlassen kann. Er wird seine Arbeit als Monteur schon in zwei Wochen gesundet wieder aufnehmen können.

\section{Fortschritte in der Behandlung des entfernbaren Pankreaskarzinoms}

Ein anschauliches Beispiel für die kontinuierlichen Fortschritte der Viszeralchirurgie in den letzten 20 Jah- ren liefert die Behandlung des Bauchspeicheldrüsenkrebses. Das duktale Adenokarzinom, welches für ca. $85 \%$ aller Bauchspeicheldrüsenkarzinome verantwortlich ist, ist die zehnthäufigste Krebserkrankung in der westlichen Welt und steht bei den krebsbedingten Todesfällen aufgrund der schlechten Prognose an vierter Stelle [2]. Die Sterblichkeitsrate entspricht nach wie vor nahezu der Auftretenshäufigkeit der Erkrankung [3, 4], da sich die Tumore bei Diagnosestellung meist in einem fortgeschrittenen, nicht entfernbaren Stadium befinden oder bereits Metastasen aufweisen. Durch die Viszeralchirurgie kann jedoch bei den Patienten mit entfernbaren Tumoren - also einem von fünf Betroffenen - eine potentielle Heilung mit einer 5-JahresÜberlebensrate von ca. 25\% erreicht werden [3, 4]. Obwohl dieser Eingriff relativ gross ist, kann er heutzutage dank stetiger Verbesserung der Operationstechniken sowie der Patientenbetreuung vor, während und nach der Operation mit einer relativ geringen Mortalität von unter 5\% durchgeführt werden [5]. Wichtig ist hierbei jedoch die Erfahrung des Zentrums, da Spitäler, die viele Pankreasoperationen durchführen, bessere Resultate sowie ein höheres Langzeitüberleben beim Patienten erzielen [6]. Da bessere Operationsergebnisse die Zeit des Spitalaufenthalts verkürzen, hat dies auch Kostenvorteile.

$\mathrm{Zu}$ den wichtigsten technischen und wissenschaftlichen Errungenschaften der letzten Jahre zählt die Optimierung der Operationstechniken. So hat sich ein Operationsverfahren etabliert, bei dem Teile des Kopfes der Bauchspeicheldrüse und des Zwölffingerdarms entfernt werden können, während gleichzeitig der Magenpförtner (Pylorus) erhalten werden kann. Dadurch erleidet der Patient eine weniger lange Operation, verliert weniger Blut und ist weniger auf Blut- 
transfusionen angewiesen als bei der klassischen Whipple-Operation, bei der auch Teile des Magens entfernt werden [7]. Da auch die Diagnostik deutlich genauer geworden ist und klare Definitionen erarbeitet wurden, bei welchen Situationen eine (teilweise) Entfernung von Bauchspeicheldrüse und umgebenden Organstrukturen angezeigt ist, profitieren Patienten heute von einer zielgerichteteren Therapie. Auch das verbesserte intensivmedizinische und schmerztherapeutische Management (z.B. Anwendung von Epiduralanästhesie) erleichtert den Betroffenen die Behandlung [4]. Zudem spielt die Anwendung von laparoskopischen Techniken in der Pankreaschirurgie eine wichtige Rolle. Diese reduzieren die Blutverluste während der Operation und ermöglichen seltenere Wundinfektionen sowie kürzere Spitalaufenthalte bei gleichwertigen onkologischen Resultaten [8].

Frau K., 66 Jahre alt, eine rüstige Witwe mit drei Grosskindern, erhält die Diagnose eines Bauchspeicheldrüsenkrebses im Pankreasschwanzbereich. Mit Hilfe von modernen gastroenterologischen und radiologischen Verfahren lässt sich exakt feststellen, dass derTumor noch keine Ableger gebildet hat und sich chirurgisch entfernen lässt. Diese Operation wird an einem Zentrumsspital laparoskopisch durchgeführt und der bösartige Tumor entfernt. Auch dank Unterstützung durch moderne intensivmedizinische und schmerztherapeutische Verfahren gestaltet sich der Verlauf nach der Operation komplikationslos, so dass Frau K. schon wenige Tage später, zwar noch etwas geschwächt, aber weitgehend beschwerdefrei, aus dem Spital austreten kann. Sie hat von der positiven Entwicklung der Viszeralchirurgie der letzten 20 Jahre profitiert und kann nun zusammen mit ihrer Familie auf Heilung hoffen.

\section{Parallele Entwicklung der modernen Onkologie}

Durch die beschriebenen Fortschritte der letzten Jahre in der Behandlung des resektablen Pankreaskarzinoms profitieren die betroffenen Patienten von deutlich verbesserten klinischen Resultaten wie geringeren Komplikationsraten, aber auch von kürzeren Spitalaufenthalten und gewinnen an Lebenszeit und -qualität. Auch die Tatsache, dass die Operation nach wie vor die einzige kurative Therapieoption darstellt, rechtfertigt ihre im Vergleich zur rein palliativen Behandlung etwas höheren Kosten [9].

Bei den medikamentösen Therapieansätzen zur Behandlung der vielen Patienten mit einem nicht entfernbaren Pankreaskarzinom rechtfertigen hingegen nach heutigem Stand die damit erzielten Ergebnisse noch nicht die hohen Mehrkosten. So weist z.B. die teure Kombinationstherapie mit Erlotinib und Gemcitabine im Vergleich zur Gemcitabine-Monotherapie einen Anstieg der Überlebenszeit um lediglich etwa zwei Wochen auf [10]. Nichtsdestotrotz darf das grosse
Potential der modernen Onkologie auch im Bereich der Therapie des Pankreaskarzinoms nicht unterschätzt und muss sogar gefördert werden, wie sowohl vielversprechende präklinische Studien an Pankreaskarzinomen als auch die Durchbrüche in der Behandlung einer Vielzahl bösartiger Krebserkrankungen (z.B. Melanom, einige Leukämiearten) gezeigt haben [11]. Um die in Studien nachgewiesenen Fortschritte in Hinblick auf ihre Relevanz für die Patienten gut einordnen zu können, haben erst kürzlich die europäischen und amerikanischen onkologischen Fachgesellschaften entsprechende Rahmenwerke publiziert. Diese quantifizieren die Überlebensvorteile onkologischer Therapieformen im Hinblick auf die Toxizitäten und Zugewinne von Lebensqualität und stellen in $\mathrm{Zu}$ kunft den Massstab für den Therapienutzen und die Kosten auch bei viszeralchirurgischen Patienten dar $[12,13]$.

\section{Ausblick: Weitere Entwicklung, ausbleibende Kostenexplosion}

Abschliessend kann festgehalten werden, dass aus der Sicht viszeralchirurgischer Patienten sowohl rein technische Innovationen als auch Fortschritte in der begleitenden medizinischen Therapie von grosser Bedeutung sind. Auch wenn eine wesentliche Kostensteigerung in diesem Bereich nicht absehbar ist, wird sich eine moderne Gesellschaft doch einen gewissen Mehraufwand leisten müssen, um ein hohes Niveau in der gesundheitlichen Versorgung sicherstellen zu können. Nicht zuletzt zur Begrenzung der Kosten sollte die Anwendung von innovativen therapeutischen Strategien jedoch stets kritisch evaluiert werden.

\section{Zusammenfassung}

Die Fortschritte der Viszeralchirurgie haben den $\mathrm{Pa}$ tienten in den letzten 20 Jahren einen klaren Nutzenzuwachs gebracht. So ermöglicht die Laparoskopie minimal-invasive und damit deutlich weniger belastende Operationen, die ein gutes Kosten-Nutzen-Verhältnis aufweisen. Bei der operativen Entfernung von Pankreaskarzinomen haben sich verbesserte Techniken und Prozesse etabliert, aber auch verbesserte intensivmedizinische und schmerztherapeutische Behandlungen kommen den Patienten zugute. Im Bereich der medikamentösen Therapieansätze sind die Erfolge zwar noch überschaubar, einige Entwicklungen lassen aber auf weitere Fortschritte hoffen, deren Relevanz für den Patienten und deren Kosten-Nutzen-Relation auch auf Basis neuer Rahmenwerke gut abgeschätzt werden kann. 


\section{Literatur}

1 Siewert JR, Rothmund M, Schumpelick V. Praxis der Viszeralchirurgie-Gastroenterologische Chirurgie (3. Auflage). Springer Verlag. 2011.

2 Siegel R, Naishadham D, Jemal A. Cancer statistics, 2013. CA Cancer J Clin. 2013;63:11-30.

3 Ryan DP, Hong TS, Bardeesy N. Pancreatic adenocarcinoma. N Engl J Med. 2014 Sep 11;371(11):1039-49.

4 Heestand GM, Murphy JD, Lowy AM. Approach to patients with pancreatic cancer without detectable metastases. J Clin Oncol. 2015 Jun 1;33(16):1770-8.

5 Winter JM, Cameron JL, Campbell KA, et al. 1423 pancreaticoduodenectomies for pancreatic cancer: A single-institution experience. J Gastrointest Surg. 2006;10:1199-1210.

6 Güller U, Warschkow R, Ackermann CJ, Schmied B, Cerny T, Ess S. Lower hospital volume is associated with higher mortality after oesophageal, gastric, pancreatic and rectal cancer resection. Swiss Med Wkly. 2017 Jul 27;147:w14473.

7 Karanicolas PJ, Davies E, Kunz R, et al. The pylorus: take it or leave it? Systematic review and meta-analysis of pylorus-preserving versus standard whipple pancreaticoduodenectomy for pancreatic or periampullary cancer. Ann Surg Oncol. 2007;14:1825-34.

8 Venkat R, Edil BH, Schulick RD. Laparoscopic distal pancreatec tomy is associated with significantly less overall morbidity compa- red to the open technique: a systematic review and meta-analysis. Ann Surg. 2012;255:1048-59.

9 O'Neill CB, Atoria CL, O'Reilly EM, LaFemina J, Henman MC, Elkin EB. Costs and trends in pancreatic cancer treatment. Cancer. 2012 Oct 15;118(20):5132-9.

10 Moore MJ, Goldstein D, Hamm J, et al; National Cancer Institute of Canada Clinical Trials Group. Erlotinib plus gemcitabine compared with gemcitabine alone in patients with advanced pancreatic cancer: a phase III trial of the National Cancer Institute of Canada Clinical Trials Group. J Clin Oncol. 2007 May 20;25(15):1960-6.

11 Vennin C, Chin VT, Warren SC, et al. Transient tissue priming via ROCK inhibition uncouples pancreatic cancer progression, sensitivity to chemotherapy, and metastasis. Sci Transl Med. 2017 Apr $5 ; 9(384)$.

12 Del Paggio JC, Sullivan R, Schrag D, Hopman WM, Azariah B, Pramesh CS, Tannock IF, Booth CM. Delivery of meaningful cancer care: a retrospective cohort study assessing cost and benefit with the ASCO and ESMO frameworks. Lancet Oncol. 2017 Jul;18(7):88794.

13 Young RC. Value-Based Cancer Care. N Engl J Med. 2015 Dec 31;373(27):2593-5. 